\title{
Potentiometrische Simultanbestimmung von Kalium und Natrium in biologischen Flüssigkeiten
}

\author{
Von H. TrutNovsky \\ Aus dem Medizinisch-Cbemischen Institut und Pregl-Laboratorium der Universität Grą
}

(Eingegangen am 29. März 1971)

\begin{abstract}
Es wird ein Gerät zu simultanen direktpotentiometrischen Bestimmung von Kalium und Natrium beschrieben. Je eine für das jeweilige Ion spezifische Elektrode und eine Bezugselektrode tauchen in einem thermostatisierten Gefäß in die mit Puffer versetzte Probe ein. Die Spannungen der zwei Meßelektroden werden einem Verstärker mit sehr hohem Eingangswiderstand zugeführt, es werden von den verstärkten Spannungen die Exponentialfunktionen gebildet und nach Korrektur für die Natriumquerempfindlichkeit der Kaliumelektrode die Konzentrationen an linear geteilten Zeigerinstrumenten angezeigt.
\end{abstract}

\section{Potentiometric method for the simultaneous measurement of potassium and sodium in biological fluids}

An apparatus is described for the simultaneous, direct potentiometric measurement of potassium and sodium in biological fluids. Specific electrodes for each ion, together with a reference electrode, are immersed into the buffered sample in a thermostated vessel. The potentials of the measuring electrodes are fed to amplifiers with high input impedance. The exponential functions are generated from the amplified potentials, the potassium result is corrected for the sodium error of the potassium electrode and the concentrations are displayed on meters with linear scales.

Bestimmungen von Kalium und Natrium in biologischen Flüssigkeiten gehören zu den Routinetätigkeiten in jedem klinischen Laboratorium. Diese Bestimmungen werden heute fast ausschließlich flammenphotometrisch durchgeführt. Bei den Möglichkeiten, die die Selektivität der modernen ionenspezifischen Elektroden bietet, ist es nicht erstaunlich, daß sie bereits frühzeitig für diese Bestimmungen verwendet wurden (1). In der Literatur beschriebene Versuche in dieser Richtung haben aber, vielleicht wegen zu aufwendiger Konstruktion $(2,3)$, keine allgemeine Verbreitung gefunden. Dabei wären Geräte auf der Basis ionenspezifischer Elektroden besonders für kleine Serien und Einzelmessungen geeignet. Selbstverständlich wird hier nur die Konzentration an freien Ionen angezeigt, bei ausreichender Verdünnung der Proben wird diese jedoch gleich der flammenphotometrisch gemessenen gesamten Ionenkonzentration (4).

Für diese Bestimmung lassen sich die interessierenden Probenmaterialien in zwei Gruppen einteilen, deren typische Vertreter Harn und Plasma sind. Bei vergleichbarem Natriumgehalt liegen die Kaliumgehalte im Plasma relativ niedrig, ganz im Gegensatz zum Harn. Außerdem beeinflußt in höheren Konzentrationen anwesendes Eiwei $\beta$ zwar nicht die Reproduzierbarkeit der Messungen, doch den Meßwert $(4,5)$. Im folgenden soll über den Bau eines Gerätes auf der Basis ionenspezifischer Elektroden und seine Anwendung zur Analyse von Harn berichtet werden. Das Problem der Analyse von Plasma mit seinem niedrigen Kalium- und hohen Eiweißgehalt soll einer späteren Mitteilung vorbehalten bleiben.

\section{Theoretische Grundlagen}

Wie eine Übersicht über die Literatur zeigt, sind für die Bestimmung von Natrium und Kalium verschiedene Elektrodentypen mit bemerkenswerten Eigenschaften entwickelt worden. Dabei ist die Selektivität der natriumspezifischen Glaselektroden $(6,7)$ so hoch, daß sie in physiologischen Flüssigkeiten rein natriumspezifisch anzeigen. Die analogen kaliumspezifischen Elektroden zeigen dagegen eine merkliche Querempfindlichkeit für Natrium und $\mathrm{H}^{+}$. Neue Entwicklungen auf dem Gebiet der Membranelektroden $(8,9)$ lassen jedoch den natriumspezifischen Elektroden gleichwertige Ausführungen erwarten.

Den Zusammenhang zwischen der Zusammensetzung der Meßlösung und der zwischen einer Bezugselektrode und den ionenspezifischen Elektroden zu messenden Spannungen (EMK) beschreibt für einwertige Kationen die von EIsEnMan (10) empirisch aus der Gleichung nach NERNST entwickelte Formel

$E M K=E_{0}+\frac{R T}{F} \ln \left[\left(A^{+}\right)^{1 / n_{A B}}+k_{A B}^{1 / n_{A B}} \cdot\left(B^{+}\right)^{1 / n_{A B}}\right]^{n_{A B}}$

Dabei sind:

$\mathrm{E}_{0}$ das Potential der Bezugselektrode bzw. der inneren Ableitungen,

$\mathrm{R}$ die Gaskonstante,

T die absolute Temperatur bei der Messung,

F die Faradaysche Konstante,

$\mathrm{A}^{+}$und $\mathrm{B}^{+}$die Aktivitäten zweier einwertiger Kationen. 
Die Größen $n_{A B}$ und $k_{A B}$ sind die von EIsENmaN in die NERNSTsche Gleichung empirisch eingeführten Koeffizienten. Bei der Messung von $\mathrm{Na}^{+}$und $\mathrm{K}^{+}$in alkalisch gepufferter Lösung ist dabei $\mathfrak{n}_{\mathrm{AB}}$ in guter Näherung gleich $1, \mathrm{k}_{\mathrm{AB}}$ ist dabei die für Elektrode und Ionenpaar charakteristische Selektivitätskonstante. Für den Fall der Messung von Natrium kann, da $\mathrm{k}_{\mathrm{NaK}} 10^{-3}$ ist, das 2. Glied in der Klammer von G1. 1 vernachlässigt werden, es ergibt sich

$$
\mathrm{EMK}_{\mathrm{Na}}=\mathrm{E}_{0}+\frac{\mathrm{R} \cdot \mathrm{T}}{\mathrm{F}} \cdot \ln \left(\mathrm{c}_{\mathrm{Na}} \cdot \mathrm{fa}_{\mathrm{Na}}\right)
$$

bzw. für die Messung von Kalium

$\mathrm{EMK}_{\mathrm{K}}=\mathrm{E}_{0}+\frac{\mathrm{R} \cdot \mathrm{T}}{\mathrm{F}} \cdot \ln \left(\mathrm{c}_{\mathrm{K}} \cdot \mathrm{fa}_{\mathrm{K}}+\mathrm{k}_{\mathrm{KNa}} \cdot \mathrm{c}_{\mathrm{Na}} \cdot \mathrm{fa}_{\mathrm{Na}}\right)$

Dabei sind:

$\mathrm{c}_{\mathrm{Na}}$ und $\mathrm{c}_{\mathrm{K}}$ die Ionenkonzentrationen,

$\mathrm{k}_{\mathrm{KNa}}$ die Selektivitätskonstante der Kaliumelektrode gegenüber Natrium und

fa die Aktivitätskoeffizienten, die den Einfluß der Gesamtionenstärke der Lösung auf die Messung berücksichtigen.

Daten der verwendeten handelsüblichen Glaselektroden zeigt Tabelle' 1 . Hier muß, um brauchbare Kaliumwerte

Tab. 1

Daten der verwendeten ionenspezifischen Elektroden, $R_{1}$ innerer Widerstand der Elektroden, $k_{i j}, k_{i} H$ und $k_{i}$ I $g$ sind die Selektivitäts\begin{tabular}{l} 
Widerstand der Elektroden, $\mathbf{k}_{i j}, \mathbf{k}_{i} \mathrm{H}$ und $\mathrm{k}_{\mathbf{i} \mathrm{Mg}}$ sind die Selektivitats- \\
konstanten gegenüber dem 2. Alkaliion, gegenüber $\mathrm{H}^{+} \mathrm{bzw} \mathrm{Mg}^{2+}$ \\
\hline
\end{tabular}

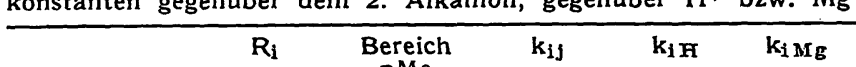

\begin{tabular}{lccccc} 
& $R_{\mathbf{i}}$ & $\begin{array}{c}\text { Bereich } \\
\text { pMe }\end{array}$ & $k_{i j}$ & $k_{\mathbf{i}}$ & $k_{\mathbf{i} \text { G }}$ \\
\hline $\begin{array}{l}\text { Na-Elektrode } \\
\text { K-Elektrode }\end{array}$ & $300 \mathrm{M} \Omega$ & $1-5$ & $1,4 \cdot 10^{-4}$ & 0,13 & $3,7 \cdot 10^{-11}$ \\
\hline
\end{tabular}

zu erhalten, wie auch aus Gl. $2 \mathrm{~b}$ ersichtlich, für den Natriumgehalt der Probe korrigiert werden. Diese Korrektur kann konventionell mittels Korrekturkurven (11), rechnerisch (12) oder, wie in diesem Gerät, elektronisch erfolgen.

$\mathrm{Da}$, bedingt durch die derzeit allgemein übliche Flammenphotometrie, die Angabe der Konzentrationen und nicht der an sich interessanteren Aktivitäten $(13,14)$ üblich ist, müssen auch die Aktivitätskoeffizienten berücksichtigt werden. Die einfache Formel von DEBYE und HÜCKEL zu ihrer Berechnung gilt leider nur für sehr verdünnte Lösungen ausreichend genau. Zur Berechnung der Tabelle 2, die den Einfluß der gesamten Ionenstärke der Lösung auf die Meßwerte zeigt, wurden daher empirische Werte (15) verwendet.

Tab. 2

Einfluß der Ionenstärke $\mathrm{J}$ auf die Aktivitätskoeffizienten fa

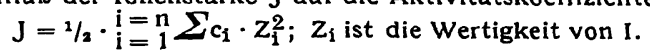

Verhältnis der Aktivitätskoeffizienten $Q ; Q=\frac{f a_{(0,2 M)}}{f_{(2}(0,1 M)}$, SkNa sind die Skalenteile Anzeige bei Einstellung auf $100 \mathrm{mit}$ der an

\begin{tabular}{|c|c|c|c|c|}
\hline Lösung & $\mathrm{J}$ & $\mathrm{fa}_{\mathrm{Na}}$ & $\mathbf{Q}$ & $\mathrm{Sk}_{\mathrm{Na}}$ \\
\hline $\begin{array}{l}0,1 \mathrm{M} \mathrm{NaCl} \\
0,2 \mathrm{M} \mathrm{NaCl} \\
0,1 \mathrm{M} \mathrm{NaCl}+0,2 \mathrm{M} \mathrm{MgCl} \\
0,2 \mathrm{M} \mathrm{NaCl}+0,2 \mathrm{M} \mathrm{MgCl}_{2}\end{array}$ & $\begin{array}{l}0,1 \\
0,2 \\
0,7 \\
0,8\end{array}$ & $\begin{array}{l}0,778 \\
0,735 \\
0,667 \\
0,662\end{array}$ & $\begin{array}{l}0,945 \\
0,993\end{array}$ & $\begin{array}{l}100 \\
189 \\
100 \\
199\end{array}$ \\
\hline
\end{tabular}

Wie die Tabelle zeigt, differieren die Aktivitätskoeffizienten $0,1 \mathrm{M}$ und $0,2 \mathrm{M}$ Natriumchloridlösung beachtlich. Wird jedoch durch Zusatz eines Elektrolyten, auf den die Elektrode nicht anspricht, die Gesamtionenstärke beträchtlich erhöht, so ist der Aktivitätskoeffizient $z$ war wesentlich kleiner, dafür aber nahezu unabhängig von der jeweiligen Natriumchloridkonzentration. Es ist also eine direkte Messung der Konzentrationen möglich, wenn außer dém $\mathrm{pH}$ auch die ionale Konzentration der Lösung weitgehend konstant gehalten wird; dazu versetzt man die Probe mit einem Puffer hoher Ionenstärke. Werden alle Messungen und auch die Eichung unter den gleichen Bedingungen durchgeführt, so kommt es nur zu einer Potentialverschiebung, die Steilheit der Elektroden wird dadurch nicht beeinträchtigt. $\mathrm{Da}$ es sich aber nur um Relativmessungen handelt, ist diese Verschiebung belanglos. Um die Ionenstärke des Puffers zu erhöhen, empfiehlt sich die Verwendung von Salzen mehrwertiger Ionen wie im Beispiel in Tabelle 2.

$\mathrm{Zu}$ berücksichtigen ist auch die Temperaturabhängigkeit der Spannungen nach G1. 2. Wenn sich auch die Temperaturkoeffizienten der inneren Ableitungen der Glaselektroden und der $\mathrm{Ag} / \mathrm{AgCl}-\mathrm{Bezugselektrode} \mathrm{kom-}$ pensieren, so ändert sich doch die Steilheit der Elektroden um etwa $0,3 \% /{ }^{\circ} \mathrm{C}$ bei Raumtemperatur. Obwohl eine elektronische Kompensation dieses Temperatureinflusses an sich möglich ist, wurde bei diesem Gerät der Weg der Thermostatisierung der Meßzelle gewählt.

\section{Aufbau des Gerätes}

Die aufgrund obiger Betrachtungen entwickelte Konstruktion gliedert sich in folgende Bauabschnitte:

1. Meßgefäß mit Elektroden (verwendet wurden die Elektroden 901.1Na und 901.1K der Fa. Polymetron, Glattbrugg-Zürich).

2. Elektronik und Anzeige.

Das Meßgefäß, das im Inneren des Gerätes eingebaut ist, muß bei möglichst geringem Volumen (etwa $5 \mathrm{ml}$ ) ein einwandfreies Eintauchen der Elektroden in die Probe ermöglichen und sich durch eine mechanische Saugvorrichtung möglichst rasch füllen und entleeren lassen. Dabei darf nach dem Entleeren. nur ein möglichst geringer Rückștand im Gefäß bleiben, und es muß auf $\pm 0,2^{\circ}$ konstant temperiert werden können.

Diese Anforderungen exfüllt die Anordnung nach Abbildung 1. Dabei sind die zwei Meßelektroden und die Bezugselektrode mit Schraubkappen in den Glasteil eingesetzt. Durch die engen Verbindungen und die große Entfernung zwischen Bezugs- und kaliumempfindlicher Elektrode ist keine Störung durch Diffusion von $\mathrm{KCl}$ während der Messung zu erwarten. Eine näherungsweise Berechnung der KCl-Diffusion (eine exakte Berechnung ist bei der verwickelten Form des Gefäßes unmöglich) exgibt einen Anzeigefehler von + 0,02 mMol K/l und Minute Meßdauer; ein Wert, der mit den Beobachtungen größenordnungsmäßig übereinstimmt und bei Einstelldauern von 5-10 Min. in fast allen Fällen vernachlässigt werden kann.

Das Mcßgefäß wird durch Ansaugen gefüllt, wozu die handbediente Pumpe in die Frontplatte des. Gehäuses eingebaut ist. Durch einen Hub dieser Pumpe werden $5 \mathrm{ml}$ Flüssigkeit angesaugt bzw. wieder ausgestoßen; bei der Entleerung wird auch die Verbindung zu einem Gummi-Handgebläse freigegẹben, womit die letzten Flüssigkeitsreste aus den Kapillaren geblasen werden können. Dadurch ist kein Spülen zwischen den Proben erforderlich. 


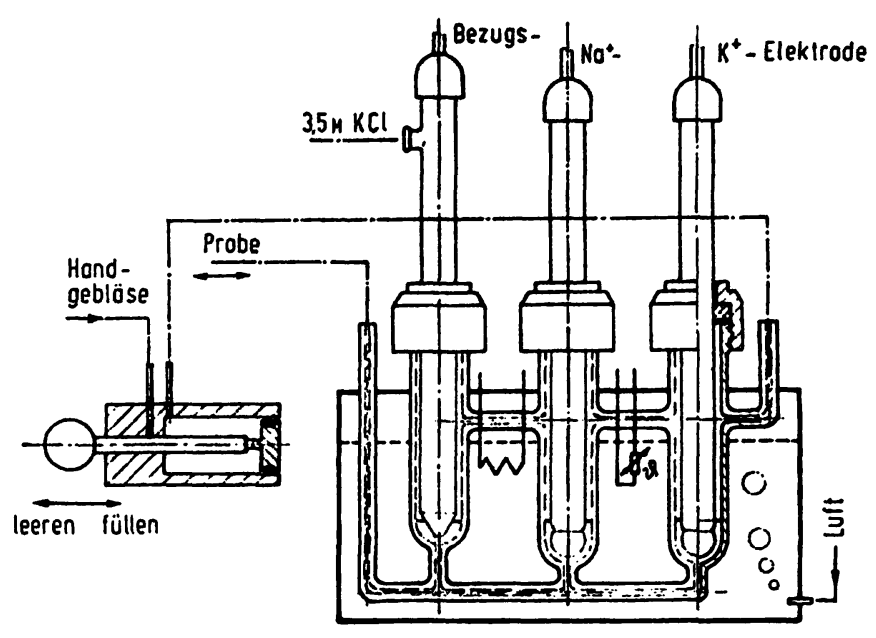

Abb. 1

Meßgefäß mit Elektroden und Füllvorrichtung

Das Glasgefäß befindet sich in einer mit Paraffinöl gefüllten Plexiglaswanne, in die ein Thermistor und ein Heizwiderstand eintauchen. Bei eincr Heizlcistung von max. $25 \mathrm{~W}$ (Proportionalregelung) werden selbst eiskalte Proben in kurzer Zeit auf die Meßtemperatur von $25^{\circ}$ erwärmt. Um eine gleichmäßige Temperaturverteilung im Thermostatenbad $\mathrm{zu}$ erziclen, wird von einer kleinen Schwingankerpumpe durch Offnungen nahe dem Boden des Bades Luft eingeblasen, die für eine rasche Durchmischung der Badflüssigkeit sorgt. Der ganze „nasse“ Teil ist als separater Einschub ausgebildet und über Steckverbindungen mit dem elektronischen Teil verbunden.

Der über zwei identische Kanäle verfügende elektronische Teil hat folgende Aufgaben:

Die von den Meßelektroden kommende Spannung ist erst durch eine Elektrometerstufe mit möglichst geringer Temperaturdrift auf kleine Ausgangswiderstände zu transformieren. Die Eingangsdaten dieser Stufe müssen mindestens den Werten eines guten $\mathrm{pH}-\mathrm{Meters}$ entsprechen. ( $R_{e} 10^{13} \mathrm{Ohm}$ und $\mathrm{I}_{\mathrm{e}} 10^{-12} \mathrm{~A}$ ). Die so erhaltene Signalspannung ist die EMK nach G1. 2. Da sie nach Abzug der Konstantspannung $E_{0}$ dem Logarithmus der gesuchten Konzentration proportional ist, muß von ihr die entsprechende inverse Funktion, die Exponentialfunktion gebildet werden. Zuvor erfolgt in einer Verstärkerstufe der Ausgleich für nicht theoretische Elektrodensteilheit, außerdem wird die Spannung auf den Arbeitsbereich des verwendeten Funktionsgenerators normiert. Die Ausgangsspannung des letzteren ist bereits proportional dem Klammerausdruck in Gl. 2. Bei der hochspezifischen Natriumelektrode kann diese Spannung direkt angezeigt werden. Im Kaliumkanal ist diese Spannung proportional $\left(c_{\mathrm{K}}+\mathrm{k}_{\mathrm{KNa}} \cdot \mathrm{c}_{\mathrm{K}}\right)$. Wird in der als Substrahierer geschalteten Nachverstärkerstufe ein entsprechender Teil der Ausgangsspannung des Natriumkanals subtrahiert, so ist die erhaltene Ausgangsspannung proportional dem Kaliumgehalt und kann angezeigt werden. Dazu dienen Drehspulinstrumente mit linearen Skalen. Eine Digitalanzeige wäre ohne Schwierigkeiten möglich, doch wäre der Aufwand unvertretbar hoch.

Diese relativ verwickelte Schaltung ist nur durch die Verwendung moderner integrierter Bauteile bei der erforderlichen Stabilität mit vertretbarem Aufwand herzustellen, ein Blockschaltbild zeigt die Abbildung 2. Auf drei gedruckten Schaltkarten (eine für jeden Meßkanal, eine für Netzteil und Thermostat) untergebracht, wird durchwegs der integrierte Operationsverstärker SN 72741 N von Texas Instruments verwendet. Es würde zu weit führen, auf alle Details der Schaltung hier einzugehen, nur zwei Stufen die von besonderem Interesse sind, sollen hier kurz besprochen werden:

Erstens das Elektrometer, Abbildung 3, das, um den erforderlichen hochohmigen Eingang zu erzielen, mit einer JFET Vorstufe ausgerüstet ist. Dafür dient der engtolerierte Dualtyp U $250 \mathrm{~A}$ von Siliconix. Der garantierte maximale Gatestrom Igss beträgt nur

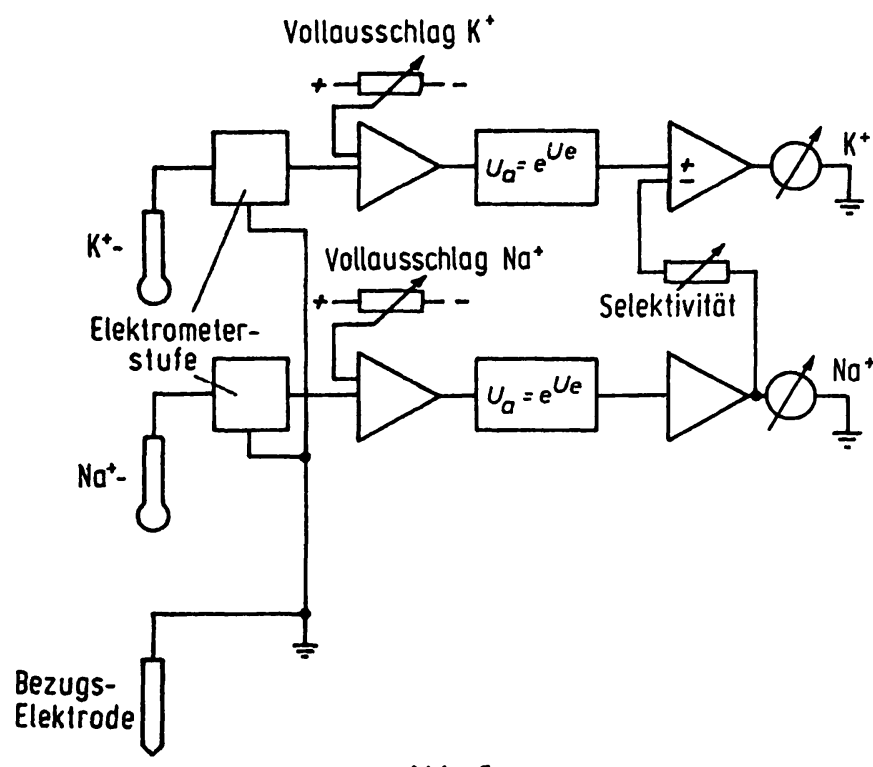

Abb. 2

Blockschaltbild der elektronischen Anordnung

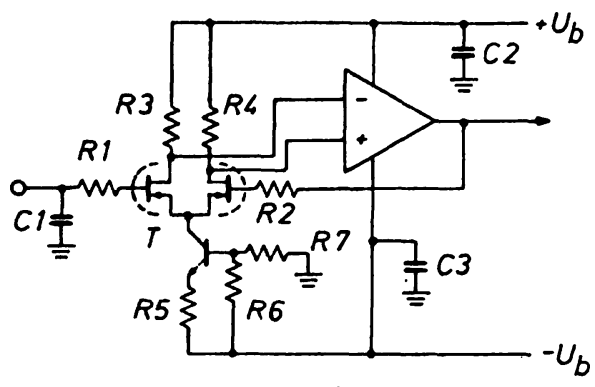

Abb. 3

Elektrometerstufe

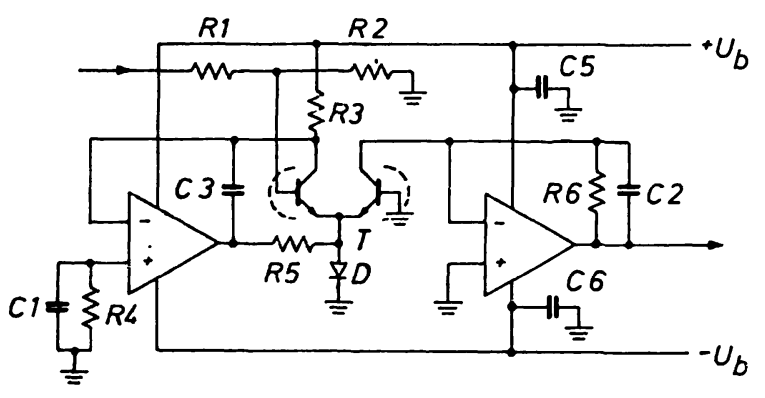

Abb. 4

Funktionsgenerator für die Exponentialfunktion

$1^{-12} \mathrm{~A}$, dic gemessenen Werte waren durchwegs um rund eine Größenordnung besser. Die Eingangsimpedanz wird durch die Gegenkopplung über den hochverstärkenden Operationsverstärker so weit erhöht, daß keine Messung mehr möglich war. Durch die Verwendung von Teflonisolation und Glaselekttodenkabeln wurden auch die Leckströme auf ein Minimum reduziert.

Die zweite bedeutsame Stufe ist der Funktionsgenerator (16) für die Exponentialfunktion, Abbildung 4. Gegenüber einer Schaltung mit einem Polygonzug aus vorgespannten Dioden hat die verwendete Ausführung mit einem Si-Planartransistor als nichtlinearem Glied den Vorteil des einfacheren Aufbaus und der präziseren Kurvenform. Zur Bildung der Exponentialfunktion wird der Zusammenhang zwischen Kollektorstrom $I_{c}$ und Basisspannung $U_{\text {be }}$ ausgenutzt. Die vereinfachte Formel dafür lautet unter der in der Schaltung zutreffenden Annahme von $U_{c e} \approx 0$ und $U_{\text {be }}>100 \mathrm{mV}$

$$
I_{c}=\alpha_{F} \cdot I_{E S} \cdot e^{\frac{-U b e \cdot q}{k \cdot T}}
$$


Hier sind:

$\alpha_{F}$ und $I_{E S}$ durch die Transistorkonstruktion festgelegte, allerdings temperaturabhängige Größen,

$\mathrm{U}_{\mathrm{cc}}$ die Kollektorspannung,

$\mathrm{q}$ die Elcmentarladung,

$\mathrm{k}$ die Boltzmann-Konstantc und

$\mathrm{T}$ dic absolute Temperatur der Transistorsperrschicht.

Durch die Verwendung eines gut gepaarten Dualtransistors kann nicht nur der Temperatureinfluß weitgehend eliminiert werden;

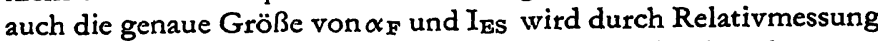
irrelevant. In einer geeigneten Schaltung wird also in sehr guter Näherung

$$
\mathrm{I}_{c}=\mathrm{K} \cdot \mathrm{e}^{-\mathrm{U}_{\mathrm{be}}}
$$

wobei

$\mathrm{K}$ ein durch die passiven Schaltelemente gegebener Proportionalitätsfaktor ist.

Um eine Beschädigung des Transistors durch eine zufällige Uberschreitung von $U_{B E O}$ zu vermeiden, ist die Diode D1 vorgesehen; es handelt sich um einen Typ (BAY 73 von SGS) mit äußerst geringem Sperrstrom, $I_{R}<5 n A$ bei $100 \mathrm{~V}$, so daß die Genauigkeit der Exponentialfunktion nicht beeinträchtigt wird. Diese Schutzschaltung ist notwendig, da bei leerer Meßzelle die Spannung von den Elektroden nicht mehr eindeutig festgelegt ist.

Besonderes Augenmerk wurde auf die Beseitigung auch der kleinsten Wechselspannungsanteile an der Signalspannung gelegt. Durch die Nichtlinearität der Exponentialfunktion würden sonst störende Anzeigefehler verursacht. Durch reichlichen Einsatz von Siebmitteln und Gleichspannungsspeisung des Thermostaten, um Einstreuungen von dieser Seite zu vermeiden, konnte die Wechselspannungskomponente des Signals auf weniger als $10 \mu \mathrm{V}$ reduziert werden.

Die Stabilität der gesamten Schaltung ist ausgezeichnet, nach kurzer Anwärmzeit liegt die Drift unter $1 \% / \mathrm{Tag}$, der Einfluß der Umgebungstemperatur bei $0,2 \% /{ }^{\circ} \mathrm{C}$. Zur Bedienung sind nur drei Einstellungen erforderlich, und zwar Vollausschlag Natrium bzw. Kalium und Selektivität. Im Routinebetrieb sind nur an den ersteren Korrekturen nötig, die Einstellung der Selektivität muß nur bei Alterung der Kaliumelektrode oder einem Wechsel der Meßbereiche verändert werden; durch eine Präzisionsskala läßt sich diese Einstellung stets wieder reproduzieren. Der normale Meßbereich, dem auch die Skalenteilung der Anzeigeinstrumente entspricht, reicht von $0-200 \mathrm{mMol} / \mathrm{l}$, wobei die Verdünnung durch den Pufferzusatz durch die Eichung bereits berücksichtigt wird. Eine Einstellung der Nullpunkte ist nie erforderlich, da sie durch den Aufbau der Schaltung automatisch stabil vorgegeben sind. Die Einstellung auf die Steilheit der verwendeten Elektroden erfolgt an zwei Einstellreglern beim Aufbau des Gerätes.

\section{Methodik}

Bestimmung der Natrium- und Kaliumkonzentration im Harn

\section{Reagenzien}

Als Puffer hat sich eine Lösung von $0,5 \mathrm{Mol}$ Triäthanolamin und $0,25 \mathrm{Mol}$ Magnesiumsulfat in Wasser, mit Eisessig auf $\mathrm{pH} 9$ gebracht und zu einem Liter aufgefüllt, sehr bewährt.

Als Eichlösungen verwendet man genau $0,2 \mathrm{M}$ Lösungen von Natriumchlorid bzw. Kaliumchlorid in Wasser.

\section{Vorgehen}

Nach dem Einschalten und einer Aufwärmzeit des Thermostatenbades von etwa $20 \mathrm{Min}$. ist das Gerät betriebsbereit.

Man überprüft mit den Eicblösungen die Vollausşchläge und stellt sie bei Bedarf nach einer Einstellzeit von 5-10 Min. nach. Dabei sind die Eichlösungen, wie dann der Harn, im Verhältnis 1:1 mit Puffer zu versetzen.

Zum Füllen der Meßzelle wird zuerst durch langsames Herausziehen des Griffknopfes der eingebauten Kolbenpumpe die bisher in der Meßzelle befindliche Flüssigkeit herausgedrückt. Ist der Knopf bis zum Anschlag herausgezogen, so sind die letzten Tropfen durch Ausblasen mit dem Gummi-Handgebläse aus den Kapillaren zu entfernen. Dann taucht man den Ansaugeschlauch in die $\mathrm{zu}$ messende Flüssigkeit und drückt den Griffknopf langsam bis zum Anschlag hinein, dadurch wird genau die zur Füllung der Meßzelle nötige Menge angesaugt. Das Mitsaugen von Luftblasen sollte vermieden werden, da Luftblasen in den Kapillaren auffallend unruhigen Zeigerstand bzw. Überschreiten der Skalenendwerte bewirken. Sie können durch kurzes, wiederholtes Betätigen der Pumpe entfernt werden.

Beim erstmaligen Gebrauch oder nach langen Arbeitspausen ist auch eine Neueinstellung der Selektivität exforderlich. Dazu wird mit Kaliumeichlösung der Vollausschlag Kalium eingestellt, dann mit Natriumeichlösung der Vollausschlag Natrium. Dabei wird durch die Querempfindlichkeit ein scheinbarer Kaliumgehalt angezeigt, er ist durch Verstellen des Selektivitätsreglers auf Null zu bringen.

Zur Messung ist der Harn wie die Eichlösungen 1:1 mit Puffer zu verdünnen. $5 \mathrm{ml}$ dieser Lösung werden zu einer Messung benötigt, die etwa 5-10 Min. dauert. Liegt ein Wert über $200 \mathrm{mMol} / 1$, so ist die Messung mit Harn, der mit Puffer 1:3 verdünnt wurde, zu wiederholen. Die abgelesenen Werte sind dann mit $2 \mathrm{zu}$ multiplizieren.

Es ist darauf $z u$ achten, daß nur frischer Harn zu den Messungen verwendet wird. Bei der Zersetzung entsteht nämlich so viel $\mathrm{NH}_{4}^{+}-\mathrm{Ion}, \mathrm{da}$ trotz der, allerdings nicht großen, Selektivität der Kaliumelektrode gegenüber Ammonium, die Kaliumwerte zu hoch erscheinen. Die physiologische Ammoniumkonzentration hat noch keinen störenden Einfluß, insbesondere da bei dem pH 9 des Puffers ein Teil des Ammoniums als undissoziiertes $\mathrm{NH}_{4} \mathrm{OH}$ vorliegt, auf das die Elektrode nicht anspricht.

Die Reproduzierbarkeit aufeinanderfolgender Messungen ohne eine Korrektur an den Einstellungen des Gerätes dazwischen zeigt die Tabelle 3. Die zu beobachtende Drift in der Größenordnung von $\pm 2 \mathrm{mMol} / \mathrm{Std}$. ist

Tab. 3

Reproduzierbarkeit der Messungen in an $\mathrm{Na}^{+}$und $\dot{\mathrm{K}}^{+}$je $0,1 \mathrm{M} \mathrm{Lö-}$ sungen bei 5 Min. Einstellzeit pro Messung

\begin{tabular}{ccc}
\hline Messung Nr. & mMol Na & mMol $\mathbf{K}^{+}$ \\
\hline 1 & 100 & 100 \\
2 & 100 & 100 \\
3 & 99 & 100 \\
4 & 99 & 99 \\
5 & 99 & 99 \\
6 & 99 & 101 \\
7 & 99 & 100 \\
8 & 99 & 98 \\
9 & 99 & 98 \\
10 & 99 & 96 \\
\hline
\end{tabular}


auf ein Abwandern der Eelktrodenpotentiale zurückzuführen, da die Schaltung selbst wesentlich stabiler ist.

Die Tabelle 4 zeigt die Ergebnisse von Messungen einer Serie von wahllos gewonnenen Harnproben mit diesem Gerät bzw. einem Flammenphotometer. Die weitgehende Übereinstimmung zeigt die Verwendbarkeit dieses Gerätes zur Harnanalyse.

Tab. 4

Vergleichsmessungen Potentiometrie-Flammenphotometrie an wahllos gewonnenen Harnproben

\begin{tabular}{ccccc}
\hline Harn Nr. & $\begin{array}{c}\text { mMol Na/1 } \\
\text { Flammen- } \\
\text { photometrie }\end{array}$ & $\begin{array}{c}\text { mMol K/l } \\
\text { metrie }\end{array}$ & $\begin{array}{c}\text { Flammen- } \\
\text { photometrie }\end{array}$ & $\begin{array}{c}\text { Potentio- } \\
\text { metrie }\end{array}$ \\
\hline & 210 & 205 & 68 & 70 \\
2 & 98 & 99 & 70 & 69 \\
3 & 66 & 54 & 46 & 48 \\
4 & 116 & 113 & 123 & 127 \\
5 & 88 & 87 & 148 & 147 \\
6 & 170 & 175 & 64 & 63 \\
7 & 143 & 144 & 52 & 52 \\
8 & 184 & 180 & 97 & 93 \\
9 & 149 & 140 & 78 & 82 \\
10 & 225 & 220 & 35 & 39 \\
\hline
\end{tabular}

Der große Vorteil dieses neuen Gerätes beruht auf der Möglichkeit, Störungen im Natrium-Kalium Haushalt auch dann zu erfassen, wenn die auffallende Konstanz des Na-K Spiegels im Blut noch keine Aussage erlaubt. So bleibt der K-Gehalt des Plasmas noch lange im normalen Bereich von 4-5 mMol/l, auch wenn die
K-Reserve der Erythrocyten durch den Kaliumverlust im Harn bereits erschöpft ist. Jede Hyperhydration ist isosmotisch und führt mit der Wasserretention zu einer vermehtten renalen Retention des extrazellulären Leitelementes Natrium, dessen Plasmaspiegel sich kaum innerhalb der Norm von $140-145 \mathrm{mMol} / \mathrm{l}$ ändert. Eine Ödemneigung, d. h. die allmähliche Zunahme von extrazellulärer Flüssigkeit aus verschiedenen klinischen Ursachen, bleibt lange unauffällig, wenn man nicht einen plötzlichen Gewichtsanstieg oder ein Mißverhältnis zwischen Flüssigkeitsaufnahme und Ausscheidung beobachtet. Es müssen bei einer normalen extrazellulären Flüssigkeitsmenge von 141 mindestens 1,51 zusätzlich einbehalten werden, soll eine sichtbare ödematöse Schwellung auftreten. Das erfordert eine renale Retention von $5 \mathrm{~g}$ Natrium, die in Form von $12,7 \mathrm{~g}$ Natriumchlorid mit der Nahrung in den Körper gelangen (Tagesbedarf). Das Elektrolytniveau bleibt bei dem Ödemkranken unverändert, und die Natriumwerte des Plasmas sind kein brauchbares Maß für das Fortschreiten, Stationärbleiben oder die Ausschwemmung eines Ödems, etwa nach saliuretischer Therapie. Im Natriumgehalt des Harnes kommen dagegen Retentionen und Ausschwemmungen stets deutlich zum Ausdruck. Daß Verschiebungen des $\mathrm{K} / \mathrm{Na}$ Quotienten im Harn ein wesentliches Merkmal für Streßsituationen darstellen, ist aus der Pathophysiologie der Nebennierenrinde hinreichend bekannt.

\section{Literatur}

1. Moore, E. W. und D. W. Wilson, J. Clin. Invest. 42, 293 (1963). - 2. Friedman, S. M. und F. K. Bowers, Analytic. Biochem. 5, 471 (1963). - 3. Portnoy, H. D., W. M. Thomas und E. S. Gurdjian, Talanta 9, 119 (1962). - 4. Friedman, S. M. und M. Nakashima, Analytic. Biochem. 2, 568 (1961). - 5. Oefme, F., G. I. T. 11,16 (1967). - 6. StefanaC, Z. und W. Simon, Analytic. Letters 1, 1 (1967). - 7. StefanaC, Z. und W. Simon, Helv. Chim. Acta 51, 74 (1968). - 8. Rechnitz, G. A., Analytic. Chem. 41, 109A (1969). - 9. ProdA, L. A. R. und W. Simon, Chimia 23, 72 (1969). - 10. Eisenman, G., D. O. Rudin und C. U. Casby, Science 126, 831 (1957). - 11. Oehme, F.,
G. I. T. 11, 1051 (1967). - 12. Jacobson, H., Analytic. Chem. 38, 1951 (1966). - 13. Dahms, H., R. Rock und D. Seligson, Clin. Chem. New York 14, 859 (1968). - 14. Jacobson, H., Ann. N. Y. Acad. Sci. 153, 486 (1968) durch Analytic. Abstr. 17, 2856 (1969). 15. Handbook of Chemistry and Physics, 46th Ed., The Chemical Rubber Co., Cleveland, Ohio USA (1965). - 16. Borlase, W. und E. David, „Design of Temperature-Compensated LogCircuits employing Transistors and Operational Amplifiers", Druckschrift der Fa. Analog Devices, Cambridge, Mass. USA (1969).
Dr. H. Trutnovsky A $8010 \mathrm{Graz}$

Universitätsplatz 2 